\title{
SALUD LABORAL EN EL ESCENARIO PRODUCTIVO ACTUAL LA CRECIENTE INCIDENCIA DE LOS RIESGOS PSICOSOCIALES
}

\author{
María Laura Henry
}

\begin{abstract}
Resumen
Los procesos productivos imponen a los trabajadores un cúmulo de exigencias con el fin de que estos brinden su mayor esfuerzo y se ajusten a las metas fijadas por la empresa u organización empleadora. Bajo estas condiciones, quedan expuestos a numerosos riesgos y a procesos de desgaste que van cambiando históricamente en función de los patrones productivos vigentes. Este artículo tiene como objetivo examinar la creciente incidencia que tiene una nueva clase de riesgos, los denominados riesgos psicosociales en el trabajo, y cómo se relacionan con el actual régimen de acumulación capitalista. Para ilustrar estas cuestiones, reseñaremos dos investigaciones realizadas en Argentina, que permiten vislumbrar las articulaciones entre la forma en la que se organiza el proceso de trabajo $y$ los riesgos $y$ enfermedades que afectan a los trabajadores.
\end{abstract}

Palabras clave: riesgos psicosociales / servicios / salud laboral / proceso de trabajo.

\begin{abstract}
Labour process and occupational health in the current productive scenario. The increasing incidence of psychosocial risks at work

The productive processes impose a series of demands on workers so that they provide their best effort and comply with the goals set by the organization that employs them. Under these conditions, workers are exposed to numerous risks and to processes of wear down that change historically, depending on the existing production patterns. The objective of this article is to examine the growing incidence of a new class of risks, called psychosocial risks at work, and how they relate to the current regime of capitalist accumulation. In order to illustrate these issues, we will review two researches carried out in Argentina, which allow visualizing the relations between the way labour process is organized and the risks and diseases that affect workers.
\end{abstract}

Keywords: psychosocial risks / services / occupational health / labour process.

\footnotetext{
María Laura Henry. Doctora en Ciencias Sociales y magíster en Ciencias Sociales del Trabajo por la Universidad de Buenos Aires (UBA). Licenciada en Sociología por la Universidad Nacional de La Plata (UNLP). Becaria posdoctoral del Consejo Nacional de Investigaciones Científicas y Técnicas (CONICET) de Argentina. Profesora de Sociología de la Facultad de Humanidades y Ciencias de la Educación de la UNLP.

E-mail: mlaurahenry@gmail.com
} 


\section{Presentación}

Desde sus orígenes, el capitalismo se modifica de manera incesante y revoluciona sus formas de producción con el objetivo de lograr un aumento de la productividad y de la rentabilidad de las empresas. Esto ha sido ampliamente analizado desde la economía crítica y otras corrientes afines, pero, en comparación, es aún reducido el corpus de estudios que se preguntan cómo estos movimientos impactan sobre los trabajadores y sus condiciones de salud. Con certeza, se trata de un tema de primera importancia que pone bajo la lupa el costo social que implican estos cambios productivos.

$\mathrm{Al}$ respecto, es importante subrayar que, en esta búsqueda incesante de ganancias y de eficiencia, las organizaciones imponen a los trabajadores un amplio abanico de exigencias, de esfuerzos y de presiones para que su productividad sea alta y constante. En estas condiciones, los trabajadores quedan expuestos a numerosos riesgos que dañan su salud, sufriendo en el camino un desgaste (físico y mental) que afecta su bienestar de maneras muy diversas y aún desconocidas.

En este artículo argumentaremos de qué forma en cada etapa de la acumulación capitalista los riesgos laborales van transformándose y hacen surgir perfiles patológicos diferentes para los trabajadores. En este sentido, nuestro objetivo es poner en discusión la creciente incidencia que en la actualidad tiene una nueva clase de riesgos, los denominados riesgos psicosociales en el trabajo (RPST), y cómo esta se relaciona con la actual etapa de acumulación capitalista.

Entre otros rasgos, el actual régimen se caracteriza por la creciente flexibilización productiva, la intensificación del trabajo, la ampliación del sector servicios y la también creciente racionalización de la producción que las empresas aplican para ganar competitividad (Neffa, 2015; Antunes, 2000). En este marco, los trabajadores ya no solo sufren las enfermedades y accidentes tradicionales (asociados a factores de riesgo físicos, químicos o biológicos), sino que se ha acrecentado de manera extraordinaria la incidencia de trastornos psíquicos y emocionales. En particular, en el sector servicios los procesos productivos exigen cada vez más que los trabajadores movilicen su creatividad, emociones, aspectos éticos, comunicativos, cognitivos, etcétera, todo lo cual acrecienta su carga global de trabajo, con consecuencias para su salud física y psíquica. 
Para ilustrar estas cuestiones, en este artículo reseñaremos dos investigaciones realizadas en Argentina sobre riesgos psicosociales, en las cuales hemos participado. Esas investigaciones se realizaron en el sector servicios y permiten establecer las articulaciones (no siempre visibles ni reconocidas) que existen entre la forma en que se halla organizado el proceso de trabajo y los riesgos y enfermedades que afectan a los trabajadores.

De esta forma, el enfoque propuesto es una superación a las explicaciones individualizadoras sobre la salud laboral y pone en evidencia que las claves explicativas y también las posibilidades de intervención (en pos de la prevención) se encuentran en el ámbito del proceso de trabajo: en su contenido y en la forma en que se halla organizado.

\section{La conceptualización de la relación entre trabajo y salud}

¿Cuál es la relación entre salud y trabajo, en el marco de un régimen de acumulación capitalista? Se trata de una pregunta clave que debemos pensar detenidamente para luego comprender cómo operan los riesgos laborales en sectores y casos específicos. Esta tarea preliminar requiere un esfuerzo orientado a elucidar toda una serie de procesos de la esfera productiva que permanecen ocultos a la observación directa y, asimismo, poner en evidencia vinculaciones que se pasan por alto en un análisis tradicional sobre riesgos laborales.

Desde un enfoque materialista crítico, debemos comenzar nuestra reflexión con una proposición central: el proceso de trabajo es uno de los determinantes principales del proceso salud-enfermedad de los colectivos humanos. Se trata de un aspecto que la corriente de la medicina social ${ }^{1}$ ha demostrado con sobrada evidencia e hizo que hoy esté muy aceptado el lugar destacado que los procesos de trabajo juegan en la configuración histórica y social de la salud-enfermedad de las poblaciones (Laurell, 1993; Blanco Gil, 1989; Laurell y Noriega, 1987).

Dada su importancia para nuestro enfoque, es importante explicitar qué entendemos por proceso de trabajo y qué aspectos permite iluminar este concepto. Al respecto, es posible afirmar que un proceso de trabajo (con independencia de lo que se produce) siempre consta de tres elementos: el objeto de trabajo (materia prima, información que se transforma y procesa), los instrumentos de trabajo (maquinaria, herramientas, tecnología) y la actividad de

1 Esta corriente surgió en América Latina en la década del sesenta y cuestionó fuertemente las limitaciones de la medicina hegemónica. Subrayó que los patrones de salud y enfermedad de la población eran expresión de la estratificación de clases y de las desigualdades en sus condiciones de vida. Esta revisión crítica de la medicina tuvo sus raíces no solo en el campo científico, sino que recibió un ímpetu decisivo de las preguntas y demandas planteadas por los movimientos sociales en esa década. Esto define a la medicina social como una corriente de pensamiento con un profundo compromiso con la resolución de los problemas de salud de las clases populares (Laurell, 1989). 
trabajo propiamente dicha, donde los trabajadores ponen en acto capacidades físicas, mentales y psíquicas. Estos tres elementos enunciados se articulan, en cada caso, según una forma específica de organización y división del trabajo, y ello con vistas a la producción de bienes, servicios o conocimientos que tienen una utilidad social porque permiten satisfacer necesidades (Noriega 1993; Laurell y Noriega, 1987; Neffa, 2015).

Ahora bien, aunque el proceso de trabajo es un proceso presente en todas las sociedades y tiempos, se concreta bajo formas históricas particulares. En este punto, es importante resaltar que, en el modo de producción capitalista en el cual vivimos, el proceso de trabajo se organiza con vistas a la generación y acumulación de ganancias (Laurell, 1993). Esto conlleva significativas derivaciones que debemos examinar con cuidado.

Ante todo, es importante comprender el proceso de trabajo como una relación social y no solo como un aspecto técnico. En particular, en el capitalismo el proceso de trabajo se desarrolla en el marco de una relación históricamente necesaria, asimétrica y conflictiva entre dos clases: los dueños de los medios de producción (empleadores) y los trabajadores. Los primeros imponen a los segundos un cúmulo de exigencias con el fin de que brinden su mayor esfuerzo durante la jornada y así generen la mayor cantidad de valor posible.

Bajo esta lógica, se renuevan de manera incesante los mecanismos de control sobre los trabajadores para optimizar la productividad, se les exige una mejora continua en la calidad y cantidad de su trabajo y se les pide que comprometan más dimensiones de sí mismos en sus empleos. En un modo de producción que funciona bajo estas demandas, no es sorpresivo entonces que los trabajadores sufran un desgaste (físico y mental) que, a corto o largo plazo, afecta su salud.

De este análisis se hace evidente que, en última instancia, las enfermedades y los accidentes laborales son expresiones específicas de la explotación capitalista, son el costo humano que implica la acumulación de ganancias (Laurell, 1978; Noriega, 1993).

Asimismo, este enfoque permite comprender la dimensión colectiva que subyace a los riesgos laborales: los problemas y patologías afectan a los grupos en función de su inserción histórica y en los procesos productivos. Al respecto, Noriega (1993) utiliza el concepto de perfil patológico para referirse a las condiciones de enfermedad y muerte que afectan a un grupo de trabajadores (definido por sus características sociales comunes) en determinado momento. Por su parte, Laurell (1993) explica que las formas concretas que tiene el capital para consumir la fuerza de trabajo se expresan en patrones especificos de desgaste que pueden detectarse a través de la frecuencia e incidencia que tienen ciertas patologías entre los trabajadores en un momento histórico dado. 
De esta forma, en el ámbito colectivo se puede relevar un conjunto de indicadores que, interpretados de forma holística y relacional, permiten conocer cómo es utilizada la fuerza de trabajo en un determinado momento de la acumulación capitalista. Algunos posibles indicadores son, por ejemplo, molestias o síntomas frecuentes, enfermedades y tipos de accidentes prevalentes, la esperanza de vida o las causas frecuentes de muerte. En todos los casos, y retomando la proposición inicial, las claves explicativas de estos fenómenos deben buscarse en el proceso de trabajo y en la forma en que este se halla organizado.

Llegados a este punto, se vuelve evidente que esta concepción sobre la relación trabajo-salud que propone el materialismo histórico cuestiona fuertemente el enfoque tradicional que hoy predomina sobre los riesgos laborales, basado en los postulados de la medicina clásica y de la ingeniería. El principal problema del enfoque dominante es que ofrece un entendimiento restringido que considera que el trabajo es un ambiente (como cualquier otro) que expone a las personas a factores de riesgo causantes de enfermedad. En este análisis nada se dice de las relaciones de explotación existentes en la relación laboral o de los procesos cambiantes de extracción del plusvalor.

Asimismo, el enfoque tradicional tiende al individualismo metodológico y a un énfasis excesivo en el nivel micro, porque el objeto de su interés son los individuos aislados o, en el mejor de los casos, pequeños grupos sin alusión alguna a la relación social productiva de la cual participan. De igual forma, es un análisis que tiende a la fragmentación analítica, porque toma los factores de riesgo (los visibles, pero no aquellos más intangibles) de forma aislada y no alcanza a verlos como expresión o "síntomas" de procesos más generales. Con ello se invisibilizan los vínculos existentes entre los procesos que ocurren a nivel micro y a nivel macro (condicionantes estructurales) derivados de la lógica capitalista y que definen la configuración que adopta la organización del trabajo en cada fase de acumulación y, por lo tanto, los riesgos asociados.

En la misma línea, Noriega (1993) explica que toda la fundamentación de la medicina del trabajo clásica está hecha sobre una posición unicausalista, según la cual cada agente produce una enfermedad determinada. Como explica Laurell (1993), la pregunta que se plantea es si "el factor de riesgo X genera la enfermedad Y, y bajo qué condiciones". No obstante, es evidente que en la actualidad los procesos de trabajo producen riesgos y exigencias que ejercen sinergia entre sí, y que se manifiestan no en una enfermedad, sino en conjuntos de patologías muy variadas, pero que tienen un origen común.

De esta manera, bajo el enfoque dominante sobre salud laboral los riesgos son analizados como hechos aislados, en el marco de un proceso de producción que se concibe como neutral, ahistórico e inmodificable. Frente a 
esto es necesario proponer un enfoque alternativo donde se visibilice que las patologías individuales son la expresión concreta de relaciones de producción antagónicas, orientadas a la creación de valor e históricamente cambiantes. Solo de esa manera se puede obtener una comprensión adecuada e integral sobre múltiples problemas que afectan hoy a los trabajadores, como veremos en el próximo apartado.

\section{El actual régimen de acumulación y los procesos de trabajo}

El análisis conceptual realizado en el apartado anterior permite extraer una proposición clave: sí existen diferentes usos de la fuerza de trabajo en cada etapa de la acumulación capitalista, por lo tanto, los riesgos laborales van transformándose y surgen patrones diferenciales de patologías.

En este sentido, es importante tener una perspectiva histórica sobre los grandes modelos de organización de los procesos de trabajo en el capitalismo y cómo han ido transformándose con el tiempo. Al respecto, la literatura económica propone una serie de regímenes de acumulación sucesivos para describir este desarrollo histórico: en primer lugar, la división técnica del trabajo, que se difundió a partir del siglo XVIII con la revolución industrial y permitió una mejora extraordinaria de la productividad; luego, la llegada de la organización científica del trabajo o taylorismo, que introdujo el estudio de tiempos y movimientos; a continuación, el fordismo adicionó la automatización de la producción y la creación de una relación salarial que estimulaba una mayor productividad de los trabajadores por medio de incentivos salariales y de su nuevo rol como consumidores de bienes masivos (Neffa, 1990).

En todos los modelos arriba mencionados, la parcialización de las tareas, la intensificación del trabajo, los mecanismos de disciplinamiento y el control rígido sobre los obreros, así como las mejoras de la productividad, se lograron a un elevado costo: el aumento de la fatiga y el sufrimiento, y un marcado deterioro de la salud de los trabajadores que predispuso el incremento de los accidentes de trabajo y de ciertas enfermedades profesionales (Neffa, 2015).

A la luz de estos problemas, a inicios del siglo XX comenzaron a surgir los primeros desarrollos conceptuales sobre salud laboral y los esquemas de prevención de los riesgos, de la mano de la medicina clásica y de la ingeniería industrial. Al ser acuñados en el marco de regímenes de acumulación donde el trabajo industrial era hegemónico, el énfasis de esos primeros abordajes estuvo puesto en la dimensión física del trabajador, en los riesgos directamente visibles (químicos, biológicos, físicos) y en un acotado número de enfermedades y accidentes.

A fines de la década del setenta, la caída de la tasa de ganancia y la consecuente crisis económica marcaron el agotamiento del modelo fordista, lo 
cual generó la transición hacia una nueva fase que se halla vigente hasta la actualidad y que ha sido denominada de manera genérica como acumulación flexible o, también, posfordismo. Esta fase no ha convergido hacia un único paradigma productivo, sino que se caracteriza por la convivencia simultánea de heterogéneas (nuevas y viejas) formas de organizar el proceso de trabajo (Antunes, 2000; De la Garza, 2013).

Así, hicieron su aparición nuevas formas de organización del trabajo formuladas explícitamente para "modernizar" la estructura vertical y rígida del fordismo, tales como el toyotismo (producción justo a tiempo y en equipos, mayor implicación de los trabajadores, énfasis en la variedad y calidad de los bienes) y la denominada especialización flexible (basada en encadenamientos productivos entre empresas especializadas). También fue notable en esos años la irrupción de las industrias intensivas en conocimiento (software, contenidos culturales, tecnologías de la comunicación, etcétera) con esquemas de producción deslocalizados, intangibles y modulares. En simultáneo, sin embargo, fueron ganando terreno otros procesos productivos poco formalizados que, en algunos casos, parecieron marcar el regreso hacia rasgos de la economía tradicional ${ }^{2}$ : producción en pequeños talleres artesanales, trabajo en establecimientos precarios y marginales, producción cuentapropista, entre otras. Y no puede olvidarse la gran expansión que ha tenido el sector servicios en estas últimas cuatro décadas, en un abanico que va desde la prestación de servicios de baja calificación (personales, de venta, de cuidado, etcétera) hasta aquellos más sofisticados (financieros, logísticos, educativos, de procesamiento de información, etcétera).

Como puede verse, estas modalidades de producir bienes y servicios vigentes en la actualidad son muy heterogéneas, pero comparten ciertos rasgos que otorgan una identidad específica a esta fase: el énfasis en la flexibilidad, la intensificación del trabajo, la desregulación de los vínculos laborales, el creciente uso de las tecnologías informáticas y de la comunicación y, fundamentalmente, la creación de valor a partir del uso de las habilidades psíquicas, emocionales y cognitivas de los trabajadores.

Las características de este nuevo régimen pronto hicieron surgir renovados problemas para la salud de los trabajadores (Neffa, 2015; Moreno Jiménez, 2011; Vogel, 2002). Padecimientos cada vez más extendidos, tales como el estrés, el burn-out (síndrome de desgaste profesional), la violencia laboral, la depresión, la fatiga crónica, los trastornos del sueño, las adicciones, entre

2 En gran parte, este renovado protagonismo ha estado vinculado a los procesos de subcontratación, al achicamiento de las empresas y a la reducción de los costos laborales que tuvieron lugar en este período como vía para recuperar las tasas de ganancia y reiniciar un nuevo ciclo de crecimiento. Esto trajo consigo un aumento de la informalidad y de la precariedad en muchas actividades económicas. 
otros, comenzaron a ser objeto de preocupación en el mundo del trabajo, empujando al mismo tiempo a un cambio de perspectiva para analizarlos.

Así, la perspectiva hegemónica de prevención de riesgos laborales lentamente fue mostrando sus limitaciones para abordar estos nuevos problemas, volviéndose evidente la necesidad de nuevos enfoques que permitan comprender cómo la dimensión psicosocial (y no solo física) de los trabajadores puede verse afectada por el trabajo en determinadas circunstancias.

\section{La creciente incidencia de los riesgos psicosociales en el trabajo}

El enfoque de los RPST constituye una perspectiva innovadora y ampliada, que se propone superar los enfoques tradicionales antes mencionados. Así, en años recientes esta corriente multidisciplinar ha comenzado a generar estudios que buscan echar luz sobre la compleja articulación entre salud y riesgos laborales. El punto de partida es que las tres dimensiones de los seres humanos - física, psíquica y mental — se movilizan en la situación de trabajo, las tres están íntimamente relacionadas y deben funcionar de manera coherente y articulada para preservar la salud. Si durante la actividad de trabajo una de ellas es vulnerada, el daño repercute también sobre las otras, generando sufrimiento psíquico y mental, además de dolor físico (Neffa, 2015).

Los estudios sobre RPST también han encontrado que en los empleos actuales la carga global de trabajo es cada vez más intensa y de naturaleza cada vez más inmaterial, cognitiva e incluso emocional. Esto les exige a los individuos mayores esfuerzos en cuanto a sus dimensiones psíquicas y mentales, lo cual tiene, a corto o mediano plazo, un impacto directo sobre su salud, tanto biológica como psíquica y mental (Neffa, 2015; Gollac, 2011). Se trata, entonces, de un nuevo patrón de desgaste de la fuerza de trabajo que debe ser analizado en toda su complejidad.

A nivel internacional, es posible encontrar un equipo de investigación muy interesante localizado en Francia, donde especialistas de las ciencias sociales del trabajo han realizado conceptualizaciones y estudios exhaustivos sobre estos nuevos riesgos (Gollac, 2011 y 2012; Coutrot y Mermilliod, 2010). Uno de sus exponentes, Michel Gollac, define a los RPST como "los riesgos para la salud física, psíquica, mental y social de los trabajadores, que son generados por las condiciones y medio ambiente de trabajo susceptibles de interactuar con el funcionamiento psíquico y mental, con impactos sobre la organización o empresa donde estos se desempeñan" (2011, p. 31). Para su estudio, Gollac ordena los diversos factores de RPST en seis grandes ejes: 1) la intensidad del trabajo y tiempo de trabajo; 2) las exigencias emocionales; 3) el grado y tipo de autonomía; 4) las relaciones sociales y relaciones de trabajo; 5) los conflictos éticos y de valores; y 6) el grado de seguridad y estabilidad de la situación de trabajo. 
La corriente de los RPST plantea que la raíz última y principal de estos riesgos psicosociales reside en la forma en que se halla organizado el proceso de trabajo (Neffa, 2015; Gollac, 2011). En este sentido, si bien sus impactos son captados a través de las percepciones de los individuos (por lo cual el trabajador tiene un papel protagónico en la detección de estos fenómenos), es en la dimensión del proceso de trabajo donde deben buscarse las causas de los problemas y donde se debe intervenir para eliminarlos. De esta forma, este enfoque es una superación de las explicaciones individualizadoras sobre los padecimientos en el trabajo, que hacen recaer sobre el trabajador la responsabilidad (ya sea por "problemas psíquicos o personales" o por una supuesta "incapacidad de adaptarse" a las exigencias laborales) ${ }^{3}$.

Como puede verse, el enfoque de los RPST enfatiza la importancia de conocer el proceso de trabajo y lo que allí sucede en tanto manifestación de procesos sociales más amplios. Al hacer estas mediaciones y recuperar la unidad entre lo micro y lo macro, permite comprender de forma integral cómo los procesos de salud y enfermedad están estrechamente ligados con los rasgos del régimen de acumulación actual.

\section{Los RPST en Argentina: primeras investigaciones y diagnósticos}

En los países desarrollados la problemática de los RPST se encuentra en la agenda de organismos de primera importancia, como la Agencia Europea para la Seguridad y la Salud en el Trabajo (2003), la Organización Internacional del Trabajo (OIT, 2016), los ministerios de trabajo de España (INSHT, 2010) y de Francia (INRS, 2013). En América Latina, se destacan las experiencias de Chile y Colombia, donde estos riesgos son monitoreados por agencias estatales de salud y trabajo (Ministerio de Salud de Chile, 2013; Ministerio de la Protección Social, 2010).

En todos estos países los informes técnicos han puesto en evidencia la magnitud económica, social y humana de los RPST. Pero en Argentina, los riesgos psicosociales generados por los procesos de trabajo quedan reducidos a la experiencia individual del trabajador, permanecen invisibilizados y ni siquiera son mencionados ni reconocidos como tales en la legislación de riesgos del trabajo (Neffa, 2017).

De esta manera, la formación e información sobre RPST que poseen los actores sociales del mundo del trabajo en Argentina permanece en niveles muy bajos. Por el lado de las empresas, aún están lejos de asumir su

3 También se suelen individualizar las posibles soluciones a estos problemas. En general, las empresas y los servicios de salud laboral recomiendan que los trabajadores se sometan a tratamientos médicos o a terapias psiquiátricas que les ayuden a eliminar o paliar los síntomas que sufren. Pero se trata de soluciones parciales y temporarias, porque las causas últimas de los problemas siguen sin ser alteradas. 
función insustituible en la prevención de los riesgos psicosociales y desconocen su responsabilidad sobre los múltiples problemas que generan estos riesgos, relegándolos a la esfera individual de cada trabajador. Por el lado de los sindicatos (salvo algunas excepciones), no se han emprendido esfuerzos sistemáticos para la medición de los RPST ni campañas masivas e integrales de sensibilización sobre su incidencia en los distintos sectores productivos.

En el marco de esta vacancia en Argentina, es importante destacar las iniciativas emprendidas desde la academia (a veces en vinculación con sindicatos) para conocer y visibilizar la incidencia de los RPST. En este sentido, nos interesa reseñar algunas investigaciones desarrolladas en universidades públicas argentinas de las que hemos participado y que han intentado generar datos y elaborar propuestas de intervención sobre esta problemática.

En concreto, vamos a describir los resultados arrojados por dos investigaciones realizadas en el sector servicios (una en el sector público y la otra en el privado), que permiten ilustrar la incidencia de estos nuevos riesgos. Comenzaremos reseñando la investigación realizada en una organización pública dedicada a la provisión de servicios sociales. A continuación, expondremos los resultados encontrados en un centro de salud privado de alta complejidad. Como argumentaremos en las siguientes páginas, ambos espacios de trabajo constituyen un terreno fecundo para estudiar las modalidades que adquieren los riesgos psicosociales en los servicios, un sector clave para el actual régimen de acumulación y donde se genera en la actualidad gran parte de la riqueza y del empleo de las economías nacionales.

\section{Los riesgos psicosociales en una organización estatal}

\section{Marco general de la investigación}

Los resultados expuestos en esta sección se basan en una investigación realizada entre los años 2014 y 2016, que tuvo como objetivo diagnosticar la incidencia de los riesgos psicosociales en una organización estatal: Administración Nacional de Seguridad Social (ANSES).

En sus orígenes, este organismo estatal se encargaba sobre todo de gestionar las jubilaciones y pensiones de los ciudadanos argentinos. Pero en la última década fue expandiendo sus funciones e incorporando la prestación de numerosos servicios: asignaciones familiares, subsidios por discapacidad, becas para estudiantes, administración de créditos de vivienda, seguros de desempleo, préstamos especiales para jubilados, entre otros. En todos los casos, se trata de servicios que apuntan a resolver problemas que aquejan a los sectores más vulnerables de la sociedad. Los ciudadanos que necesitan algunas de estas prestaciones pueden acudir de manera presencial a las ofi- 
cinas de ANSES que se hallan emplazadas en todo el país y también pueden gestionar los servicios por vía telefónica.

La investigación surgió a partir de la solicitud de un sindicato con representación en la institución: el Sindicato de Empleados de la Ex-Caja de Subsidios Familiares para el Personal de la Industria (SECASFPI). Del sindicato se acercaron al equipo interdisciplinario al cual pertenecemos expresando la necesidad de un estudio que avalase científicamente una serie de problemas y malestares que afectaban a los trabajadores de ANSES, pero que las autoridades del organismo se negaban a reconocer.

En particular, desde el sindicato se percibía que los trabajadores en puestos de atención al público eran quienes manifestaban sentirse más "desbordados" por la gran afluencia de público a las dependencias. Estos expresaban malestares tales como estrés y fatiga, y una diversidad de dolencias crónicas que identificaban como efecto de su trabajo. Fue por ello que, en términos metodológicos, la investigación se circunscribió desde el inicio a esa franja de trabajadores de atención al público, dado que era prioritario conocer su situación para solicitar medidas de intervención a las autoridades en el corto plazo.

Para alcanzar los objetivos enunciados, se utilizó un abordaje metodológico que combinó técnicas cualitativas y cuantitativas de recolección de información: talleres de visualización (desde el enfoque de la psicología del trabajo); estudios de ergonomía; un estudio de medicina del trabajo y una encuesta de RPST ${ }^{4}$.

Para el análisis que presentamos en este artículo utilizamos como insumo principal los datos arrojados por la encuesta ${ }^{5}$, que permitió relevar la percepción de los trabajadores sobre distintos factores de riesgo psicosocial. Específicamente, se realizaron 694 encuestas en oficinas de ANSES de distintas ciudades de Argentina. El formulario fue administrado en formato impreso, de manera presencial, por encuestadores capacitados para tal fin, y la duración promedio de cada aplicación fue de entre 40 y 50 minutos. En todos los casos, los trabajadores participaron de manera voluntaria y anónima.

4 Para conocer en detalle la metodología general de la investigación, se sugiere ver Neffa, Korinfeld y Henry (2017).

5 El formulario usado estuvo basado en una encuesta diseñada en Francia, donde un equipo de expertos desarrolló este instrumento para relevar los RPST en la población trabajadora de aquel país. Como forma de retomar esos desarrollos, el doctor Julio C. Neffa y sus equipos de varias universidades nacionales se abocaron a examinar las condiciones de aplicación al contexto local. Fruto de una cuidadosa reflexión metodológica, lograron la confección del Cuestionario sobre riesgos psicosociales aplicable en Argentina, que se ha usado en las investigaciones que reseñamos en este artículo, así como en otras similares. Para conocer más detalles de la encuesta, véase Henry (2016). 


\section{El problema de la intensificación}

Durante la investigación realizada en ANSES se abordaron muchos aspectos del proceso de trabajo y en el diagnóstico se constató la existencia de diversos factores de riesgo psicosocial en los puestos de atención al público. Por cuestiones de espacio, los enumeraremos aquí sintéticamente: alta intensidad en los ritmos de trabajo; importante incidencia de la carga mental; fuertes exigencias emocionales por el contacto directo con personas en situación de sufrimiento y vulnerabilidad social; situaciones de violencia verbal y agresiones; falta de capacitación y formación para la resolución de las tareas que provoca en los trabajadores una autonomía forzada y padecida; falta de reconocimiento por parte de la organización (ausencia de evaluaciones y de posibilidades de carrera); insuficiencia en actividades de prevención y formación en riesgos del trabajo; entre otros aspectos.

En el marco de este conjunto de RPST detectados, quisiéramos centrarnos en uno de ellos, que emergió con especial incidencia en este colectivo de trabajadores: la intensificación del trabajo.

En términos específicos, el concepto de intensificación se refiere al aumento de la cantidad de bienes y servicios producidos, sin que se cambie la dotación de la fuerza de trabajo ni la duración de la jornada. Dicho aumento surge, entonces, de un incremento en el ritmo de trabajo o de una disminución de la porosidad de la jornada, eliminándose así los tiempos "muertos" (Neffa, 1990; Coriat, 1991).

El fenómeno de la intensificación merece una especial reflexión para el sector de los servicios, porque las presiones que reciben los trabajadores para aumentar los ritmos de trabajo provienen de dos esferas (Go1lac, 2005; Bernard, 2005; Henry, 2017). Por un lado, surgen de la esfera interna de la organización, a través de las estrategias de gestión y de producción allí vigentes. Desde esta esfera, los ritmos se hallan impuestos por el control de los superiores jerárquicos, la fijación de normas de producción, la cadencia impuesta por maquinarias y equipos, el pago por productividad, entre otras.

Por otra parte, en los servicios, la cadencia del trabajo también se halla regida desde la esfera externa a la organización, a partir de la demanda. En este plano, la afluencia de clientes o de público ejerce una presión sobre el proceso de trabajo por medio de su presencia en los espacios laborales (largas filas de gente, salas de espera llenas, etcétera), demandando a los trabajadores un cierto ritmo de trabajo. Las organizaciones y empresas cada vez más someten a los trabajadores a la acumulación de estos dos tipos de exigencias de ritmo y, en este marco, se hace necesario interrogarse sobre las consecuencias que ello tiene sobre los trabajadores y su salud. 
En el caso de la ANSES se pudo verificar la existencia del fenómeno de intensificación y los datos de la encuesta así lo demuestran. En primer término, se les consultó a los trabajadores si estaban obligados a apurarse (Gráfico 1) y las respuestas obtenidas fueron las siguientes:

\section{Gráfico 1. ¿Está usted obligado a apurarse en su trabajo?}

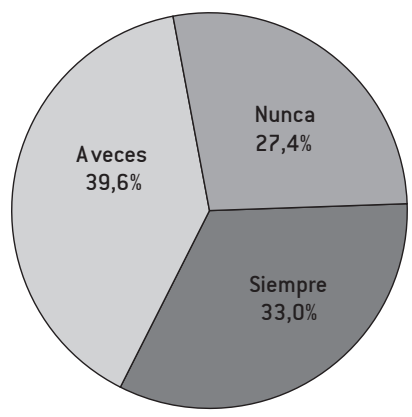

Fuente: elaboración propia.

De estos datos se desprende que - de manera agregada - un $72,6 \%$ de los encuestados dijo que "siempre" y "a veces" tiene que apurarse en su trabajo. Solo un 27,4\% indicó no tener este inconveniente.

También se consultó a los trabajadores si tenían el tiempo suficiente para realizar su trabajo (Gráfico 2):

\section{Gráfico 2. Para realizar correctamente su trabajo, ¿usted tiene en general el tiempo suficiente?}

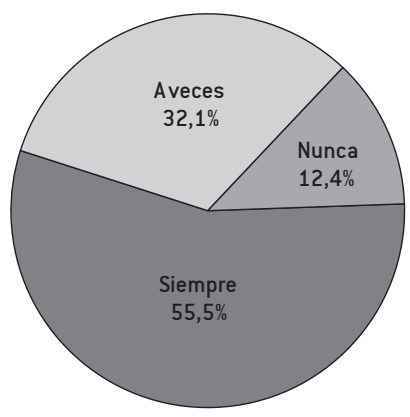

Fuente: elaboración propia.

Solo el 55,5\% dijo tener el tiempo suficiente para realizar su trabajo. Con certeza, esto indica que gran parte de los trabajadores (45,5\% si adicionamos a quienes respondieron "nunca" y "a veces") siente que necesitaría márgenes temporales mayores para desempeñar su labor. 
En el mismo sentido apunta la siguiente pregunta relevada (Gráfico 3):

Gráfico 3. ¿Con qué frecuencia tiene que hacer demasiado rápido una operación que requeriría más tiempo y cuidado para ser bien hecha?

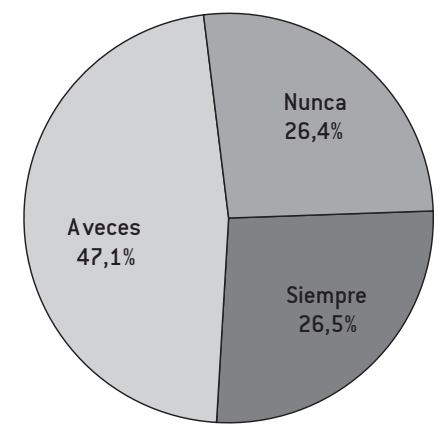

Fuente: elaboración propia.

En el Gráfico 3 puede apreciarse que el 73,6\% indicó que "siempre" o "a veces" tiene que hacer demasiado rápido una operación que requeriría más tiempo y cuidado para ser bien hecha. Solo un cuarto de ellos negó vivir esta situación $(26,4 \%)$.

En otra pregunta de la encuesta, se consultó a los trabajadores si sentían que trabajaban bajo presión, lo cual sintetiza en gran medida su percepción sobre los ritmos de trabajo (Gráfico 4):

Gráfico 4. ¿Con qué frecuencia siente usted que trabaja bajo presión?

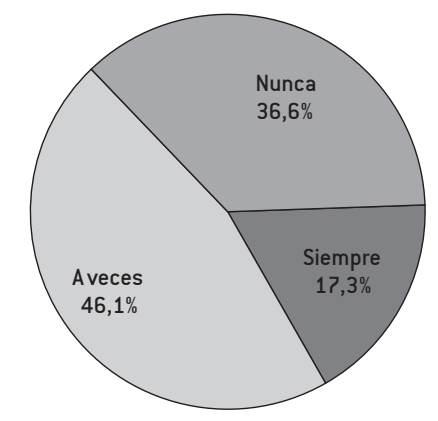

Fuente: elaboración propia.

Como se puede ver en el Gráfico 4, solo el 36,6\% de los encuestados dijo no trabajar nunca bajo presión. El resto expresó sufrir este problema: un $17,3 \%$ dijo que "siempre" le sucede y un $46,1 \%$ que le sucede "a veces".

De los datos hasta aquí presentados, surge que los trabajadores de atención al público encuestados efectivamente tenían altos ritmos de trabajo en ANSES. Esto también fue corroborado por los talleres cualitativos, donde de 
manera reiterada los trabajadores hicieron referencia a la enorme cantidad de trámites que debían resolver por día y al gran caudal de público que debían atender durante su jornada laboral, reduciendo para ello las pausas al mínimo y acelerando su velocidad de trabajo. Los relatos expresaban situaciones de agobio, remarcaban la imposibilidad de tomarse descansos e, incluso, las grandes dificultades para dejar unos pocos minutos el puesto (por ejemplo, para ir al sanitario), dado que el propio público ejerce una vigilancia sobre su labor y comienza a quejarse o a expresar su enojo verbal.

Resulta también interesante examinar cuáles son los factores que demarcan esos ritmos de trabajo en la organización. La encuesta permitió establecer que los tres principales son los que se muestran en el Gráfico 5.

Gráfico 5. Incidencia de las diferentes exigencias de ritmo (respuestas a la pregunta: ¿Su ritmo de trabajo está dado por...?]

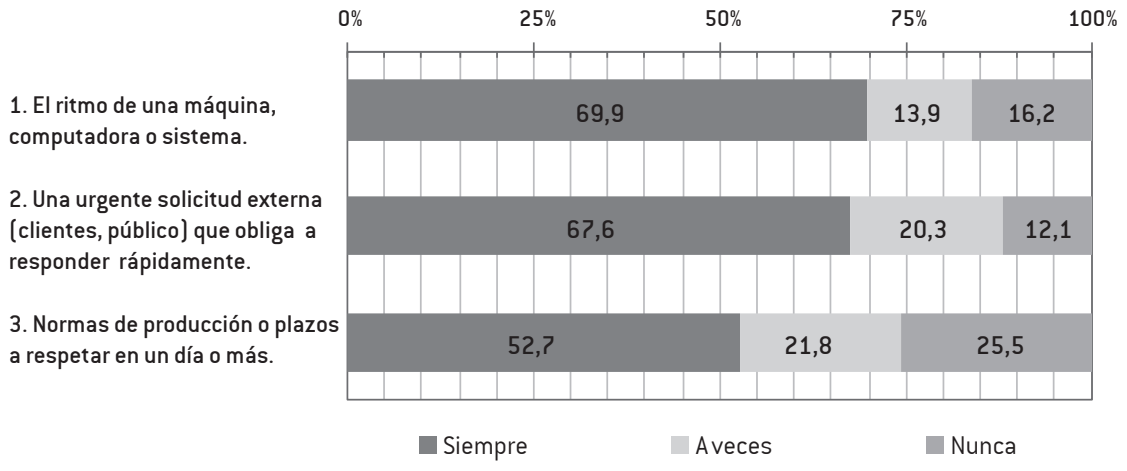

Fuente: elaboración propia.

En el Grafico 5 la primera barra nos indica la incidencia que tienen las exigencias de tipo tecnológico y técnico en los ritmos de trabajo. En ANSES, esto se materializa en los sistemas de turnos electrónicos, los sistemas telefónicos que gestionan el flujo de llamadas entrantes y los programas informáticos que se utilizan en las computadoras de la ANSES para realizar las gestiones administrativas. En todos los casos, se trata de sistemas que fijan una determinada cadencia para los trabajadores. A continuación, el Gráfico 5 muestra el rol destacado que juega la presencia del público en las dependencias de ANSES, que ejerce una gran presión sobre los trabajadores, al igual que los incontables llamados telefónicos diarios. En este punto vemos cómo la demanda externa tiene un rol importante sobre los ritmos internos de la organización. En tercer lugar, se visualiza la gran incidencia que tienen las normas y plazos en los ritmos de trabajo. En la ANSES estos objetivos de productividad hacen referencia a una cantidad de trámites o llamados telefó- 
nicos a resolver en cierto lapso de tiempo (por día o por mes) que constituyen métricas de productividad establecidas desde la gerencia.

Lo relevante, en función de nuestros objetivos, es reflexionar sobre las consecuencias que todo esto tiene sobre los trabajadores. La literatura explica cómo la intensidad puede degradar las condiciones de trabajo y afectar la salud de los trabajadores. Las exigencias de ritmo obligan a trabajar de la "forma más rápida", que no tiene por qué ser la más adecuada a las características fisiológicas y psicológicas de cada trabajador (Gollac, 2005). En el caso de los servicios, los trabajadores, asimismo, deben encontrar un compromiso entre las exigencias surgidas desde el interior de la organización y aquellas provenientes de la demanda externa. Esto constituye una doble fuente de tensiones y contradicciones que debe arbitrar con mucho esfuerzo, con el consecuente desgaste y los riesgos que ello implica.

Respecto de esto último, es importante señalar que la alta intensidad en los ritmos de trabajo potencialmente conlleva una serie de problemas para la salud de los trabajadores: incremento de la fatiga, alteraciones emocionales (ansiedad, nerviosismo, angustia), aumento de la frecuencia cardíaca, predisposición a desarrollar un cuadro de estrés crónico, entre otras derivaciones. En este sentido, el ritmo de trabajo constituye un aspecto clave en el cual debería intervenirse para prevenir la aparición de estas consecuencias negativas para la salud.

\section{Los riesgos psicosociales en un centro de salud}

\section{Marco general de la investigación}

En esta sección presentaremos los lineamientos principales de una investigación realizada en una clínica privada ${ }^{6}$, en el marco de un proyecto de desarrollo tecnológico y social cuyo equipo de trabajo estuvo compuesto por investigadores, docentes y alumnos de diversas disciplinas (economía, administración, psicología y sociología) ${ }^{7}$. Asimismo, cabe señalar que este proyecto fue desarrollado en simultáneo en distintas universidades públicas de Argentina y conllevó la interacción con distintas organizaciones de la sociedad civil, las cuales asumieron el rol de "adoptantes" de los resultados. De esta forma, el proyecto apuntó a transferir herramientas y conocimientos a la sociedad que permitan la evaluación, intervención y prevención de los riesgos psicosociales en el trabajo.

6 Por razones de confidencialidad no usaremos el nombre real de la institución y nos referiremos a ella con esta denominación.

7 Proyecto de Desarrollo Tecnológico y Social PDTS-CIN n. ${ }^{\circ} 422$ "Los riesgos psicosociales en el trabajo. Relevamiento de la percepción y vivencias por parte de los trabajadores asalariados con vistas a su prevención", dirigido por L. Ferrari y codirigido por Julio C. Neffa. 
La investigación fue desarrollada por un equipo localizado en la Universidad Nacional de La Plata (UNLP). En este caso, la organización potencialmente adoptante de los resultados fue la Asociación de Trabajadores de la Sanidad (ATSA-La Plata). Este sindicato expresó su interés en colaborar con el estudio dado que podría obtener así datos que le permitirían conocer las condiciones de trabajo de sus representados, sus principales problemas y los aspectos en los que sería necesario intervenir. De igual forma, las autoridades y propietarios de la clínica mostraron interés en conocer los resultados y en utilizarlos para mejorar la gestión de su personal.

En cuanto a sus características, la clínica privada se encuentra ubicada en la ciudad de La Plata y provee una multiplicidad de servicios de salud: atención primaria y de urgencias, internación, cirugía, cuidados de embarazo y maternidad, análisis de laboratorio, radiología, etcétera. Si nos centramos solo en el personal en relación de dependencia, la organización emplea a casi cuatrocientos trabajadores y a sus instalaciones acude a diario un gran número de pacientes y sus familiares de toda la región, siendo así un centro de salud de gran importancia local.

Para relevar los factores de riesgo psicosocial existentes, se realizó una encuesta (la misma que se utilizó en ANSES) a una muestra de ochenta trabajadores durante el segundo semestre de 2016. La selección de los trabajadores se construyó atendiendo a las características y particularidades de los procesos de trabajo realizados en el centro de salud. De esta forma se procuró que estuvieran representadas las diferentes categorías profesionales, los distintos turnos de trabajo y las diferentes áreas operativas. El formulario fue aplicado de forma presencial por encuestadores en el lugar de trabajo y fue respondido de manera voluntaria y anónima ${ }^{8}$. Asimismo, se realizó observación participante en las instalaciones y se desarrollaron entrevistas cualitativas con informantes clave (autoridades de la clínica, representantes sindicales, jefe de personal, entre otros).

\section{Las exigencias emocionales como factor de riesgo}

El sector salud es un espacio donde el estudio de los riesgos psicosociales resulta muy pertinente, dada la naturaleza de las actividades que allí se realizan. En este sentido, se trata de espacios donde los trabajadores deben movilizar no solo funciones físicas, sino fundamentalmente sus dimensiones mentales, emocionales y psíquicas de primera importancia. Día a día se ven interpelados por demandas múltiples y simultáneas, tareas complejas, emergencias y el contacto con pacientes y público, con las exigencias que ello implica (situaciones de sufrimiento, de angustia e, incluso, de agresión).

8 Para conocer más detalles sobre la metodología de la investigación, se sugiere ver Neffa y Henry (2017). 
$\mathrm{Al}$ igual que en la investigación en ANSES, durante el estudio en la clínica privada se abordaron múltiples aspectos y el diagnóstico abarcó diversos factores de riesgo psicosocial. En síntesis, los principales problemas detectados fueron: alta incidencia de la carga mental; prolongadas jornadas laborales; fuertes exigencias emocionales por el contacto directo con pacientes y familiares en situación de sufrimiento; falta de reconocimiento por parte de la organización (escasas posibilidades de carrera y salarios bajos); escaso apoyo técnico por parte de superiores; conflictos entre categorías profesionales, entre otros aspectos.

Teniendo en cuenta este cuadro de situación, aquí nos gustaría centrarnos en uno de los riesgos que devino central durante la investigación: las exigencias emocionales.

Las exigencias emocionales se refieren a la necesidad de los trabajadores de dominar y moldear las propias emociones, en especial aquellas que sienten en relación con las personas con las que interactúan en la situación de trabajo (Gollac, 2011; Neffa, 2015). Este control de las emociones a veces es requerido, ya sea tácita o explícitamente ${ }^{9}$, durante el proceso de trabajo para el logro de distintas metas: premios salariales, aumento en los niveles de ventas, el bienestar del cliente o paciente, el cumplimiento de normas de calidad o de estándares profesionales, etcétera. En otros casos, puede suceder que los trabajadores deban esconder o negar sus propias emociones por miedo a los accidentes, a la agresión del público o al fracaso de su actividad.

Como señalan Galeano y Ruiz (2017), es preciso pensar la especificidad que adquieren las exigencias emocionales en el sector de la salud. Al respecto, el cuidado de la salud tiene una significación y una valoración social particular, pues implica la preservación de la vida humana. Asimismo, sus prácticas laborales implican reconocer la vulnerabilidad y la necesidad de cuidado a las que está sometida cualquier persona. De esta forma, un conjunto de normativas, creencias y valores que apela a un compromiso con la salud y la vida se pone en juego directa o indirectamente en cada puesto de trabajo $^{10}$.

9 Hay casos donde las empresas enfatizan el uso de las emociones y prescriben formas específicas de comportamiento a los trabajadores en su relación con los clientes por medio de capacitaciones y de protocolos de interacción. Así, les indican cómo saludar, qué registro (formal o informal) usar en los diálogos y el despliegue de una serie de actitudes en su trabajo (buen humor, empatía, trato amable, disponibilidad, etcétera).

10 Para emprender un estudio de las exigencias emocionales en el sector salud es conveniente articular el análisis con, al menos, otros dos campos conceptuales: la corriente que estudia los trabajos "de cuidado" y el enfoque de género, dada la notable división del trabajo por género que aún persiste en este sector. Lamentablemente no podemos desarrollar esta articulación aquí por falta de espacio, pero para ahondar en esta temática se sugiere ver Galeano y Ruiz (2017) y Esquivel y Pereyra (2017). 
En cuanto a los datos arrojados por la investigación, en primer término, podemos señalar que en la clínica privada se detectó un alto porcentaje de encuestados que, como parte de sus tareas habituales, está en relación directa con el público (alrededor del 80\%), ya sean pacientes o sus familiares. Por sí mismo, esto demanda a los trabajadores conductas específicas, expresiones acordes con los estándares de la organización y de su profesión, así como formas de comunicación enmarcadas en ciertos parámetros.

La encuesta arrojó una serie de resultados que podemos sistematizar en dos grandes grupos de exigencias emocionales que enfrentan los trabajadores en función del tipo de trabajo que se realiza en la clínica: por un lado, exigencias vinculadas a contener a pacientes y familiares en situación de angustia $y$, por otro, exigencias vinculadas a contener las reacciones negativas del público (enojo, agresión).

Respecto de lo primero, el trato diario con población que sufre distintos problemas de salud se convierte en el punto de encuentro con múltiples formas de sufrimiento y en un lugar de escucha y de exposición de problemas privados de los pacientes y sus familias. En este marco, es comprensible que para muchos trabajadores las exigencias emocionales alcancen un alto grado al encontrarse de manera constante expuestos a estos fenómenos, como se puede ver en los Gráficos 6 y 7.

\section{Gráfico 6. Mientras trabaja, ¿está en contacto con otras personas en situación de angustia?}

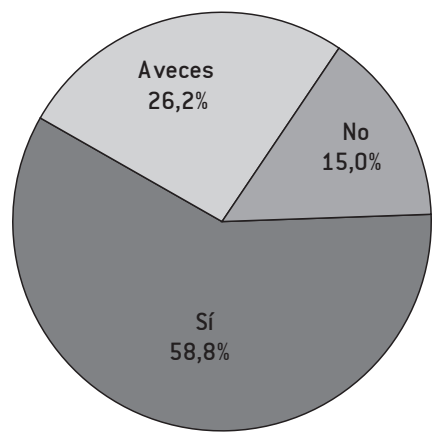

Fuente: elaboración propia.

A menudo, los procedimientos sanitarios (e incluso los trámites) que se gestionan en la clínica implican recursos vitales para los receptores. Y esto puede dar lugar a tensiones e incluso confrontaciones en la relación entre, por un lado, los trabajadores y, por otro, los pacientes y sus familiares. Esto sucede, por ejemplo, cuando estos últimos se encuentran bajo desesperación en situaciones de urgencias (accidentes, descompensaciones), en el marco de 


\section{Gráfico 7. Mientras trabaja, ¿usted debe calmar a personas angustiadas, preocupadas, nerviosas, coléricas?}

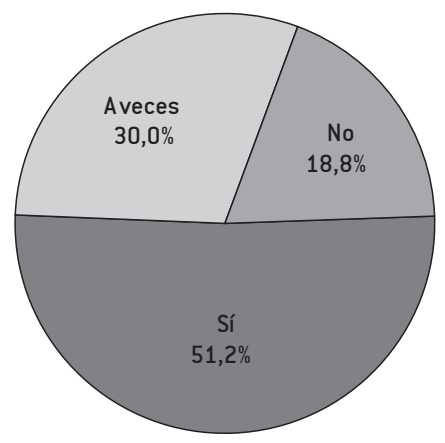

Fuente: elaboración propia.

enfermedades complejas que generan gran sufrimiento y frustración que es descargada sobre los trabajadores, o cuando se necesita con celeridad algún procedimiento sanitario que se demora por cuestiones operativas o administrativas. Al respecto, en la encuesta se les preguntó a los trabajadores si sufrían situaciones de tensión en sus relaciones con el público. Como vemos en el Gráfico 8, de forma agregada (si sumamos "siempre", "casi siempre" y "a veces"), el 42,5\% debe afrontar este tipo de problema con cierta recurrencia.

\section{Gráfico 8. ¿Sufre usted situaciones de tensión en sus relaciones con el público?}

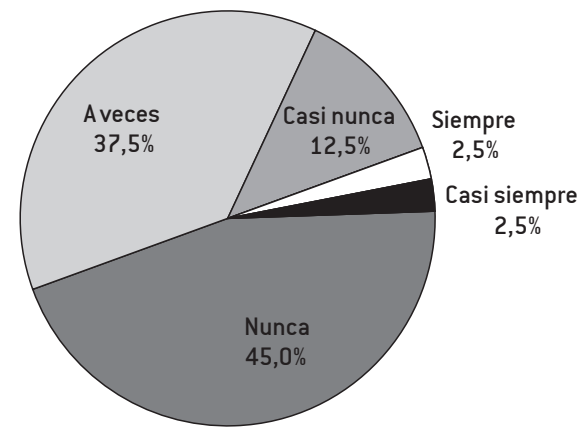

Fuente: elaboración propia.

Estas situaciones de tensión se expresan cotidianamente en los lugares de atención por medio de actitudes de impaciencia, indignación y protesta por parte del público. Y cuando ocurren estos eventos, la regulación de las propias emociones puede volverse una exigencia muy fuerte para los trabajadores. Estos se encuentran limitados por prescripciones organizacionales que les dictan pleitesía, amabilidad y la obligación de "contenerse" ante cualquier tipo de interpelación agresiva. Como se puede ver en el Gráfico 9, los 
trabajadores sobrellevan una serie de exigencias emocionales ligadas a gestionar sus estados de ánimo y producir emociones correctas para interactuar con pacientes o familiares.

\section{Gráfico 9. ¿Con qué frecuencia tiene que esconder sus emociones o fingir estar de buen humor?}

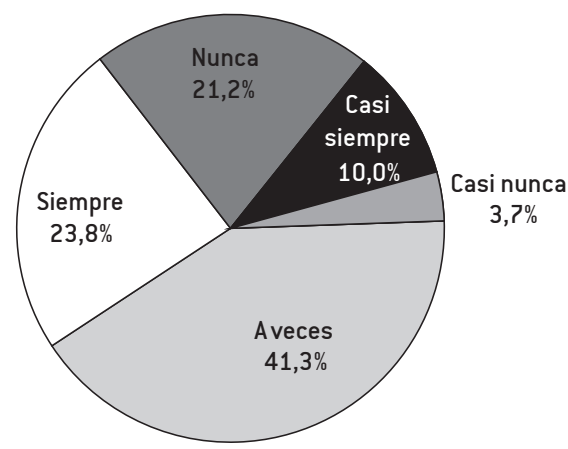

Fuente: elaboración propia.

En el gráfico se puede observar que, de forma agregada (si sumamos "siempre", "casi siempre" y "a veces"), tres cuartos de los trabajadores deben esconder sus emociones o fingir estar de buen humor con cierta recurrencia.

Los datos aquí expuestos deben ser interpretados a la luz de un proceso de trabajo que, como decíamos antes, tiene como objeto algo tan sensible como la vida humana y el bienestar de las personas. Así, en un contexto donde el sufrimiento, el dolor, la angustia e incluso la muerte son aspectos recurrentes del proceso de trabajo, es esperable que las exigencias emocionales alcancen un nivel altísimo para los trabajadores.

Esta dimensión emocional del trabajo moviliza un conjunto de procesos psíquicos de los trabajadores que contribuye a su carga global de trabajo y que, en determinadas circunstancias, puede generar sufrimiento y problemas de salud. De esta forma, la literatura identifica una serie de síntomas asociados a estas exigencias: trastornos de ansiedad, cuadros de estrés agudos, burn-out y somatizaciones físicas que conducen a enfermedades de diversa índole (Martínez Íñigo, 2001; Gracia, et al., 2007).

\section{Conclusiones}

En este artículo nos propusimos visibilizar relaciones y procesos que, en general, son ignorados por los estudios tradicionales sobre riesgos laborales. Estos proveen una perspectiva restringida, porque siguen demasiado apegados al nivel micro, recurren a explicaciones unicausales y hacen una interpretación fragmentada de la realidad. 
Como alternativa, hemos optado por revisitar el problema de los riesgos laborales desde el enfoque crítico, holístico e histórico, para alcanzar una comprensión más amplia sobre sus causas y consecuencias. La primera ventaja que surge de este tipo de análisis es que permite comprender cómo operan los determinantes sociales en los procesos de salud y enfermedad en el trabajo. De esta forma, es posible entender los riesgos laborales como un proceso dinámico y colectivo, en el que determinados grupos sociales quedan expuestos a su incidencia en el marco de modos de acumulación específicos.

En relación con esto último, hemos intentado identificar todas las consecuencias que conlleva, en la actualidad, estar inmersos en un modo de acumulación donde el proceso de trabajo está orientado a la acumulación de ganancias. Que la meta última sea esa, hace que los procesos de trabajo adquieran rasgos específicos que afectan la salud de los trabajadores.

En este sentido, hemos resaltado que las enfermedades y los accidentes laborales son expresiones específicas de la acumulación capitalista. Son el precio humano que se paga en un sistema que prioriza el lucro por sobre el bienestar y la seguridad de los trabajadores, donde las pausas y descansos son consideradas una pérdida de dinero, donde los mecanismos de control con frecuencia impiden a los trabajadores operar de la forma más adaptada a sus capacidades, donde las máquinas rigen los ritmos y donde las empresas niegan de manera sistemática muchísimos problemas de salud vinculados al trabajo para evitar el pago de indemnizaciones o para evitar modificar sus métodos de producción.

Asimismo, hemos argumentado que en cada etapa del capitalismo los diferentes usos de la fuerza de trabajo han llevado a la configuración de perfiles patológicos diferenciales dentro de la población trabajadora. En este sentido, nuestro objetivo fue poner en discusión la creciente incidencia que hoy tienen los denominados riesgos psicosociales en el trabajo y cómo se relacionan con el actual régimen de acumulación.

Para ello hemos reseñado dos investigaciones sobre este tema realizadas en Argentina, que se llevaron adelante en el sector servicios, y sus resultados permiten ver cómo las exigencias y cargas laborales propias de este sector pueden generar enfermedades y sufrimientos poco reconocidos.

En el caso de la investigación en ANSES hemos analizado el problema de la intensificación, a partir de la gran incidencia que este factor mostró tener en dicha organización estatal. Al respecto, describimos el aumento de los ritmos de trabajo, la insuficiencia de pausas y descansos y la multiplicación de tareas que debieron afrontar los trabajadores en el marco de una expansión de funciones de la organización. Todo ello es percibido como muy problemático por los trabajadores e implica riesgos potenciales para su salud y bienestar psicofísico. 
El fenómeno detectado en esta investigación es plausible de ser encontrado en muchas organizaciones (públicas y privadas), dado que el progresivo aumento de la intensificación del trabajo es una de las tendencias productivas que marca nuestra época. Producir más cantidad y más rápido se impone como el objetivo supremo desde las organizaciones en la búsqueda de mayores niveles de eficiencia (Gollac y Volkoff, 1996; Green, 2004). En este sentido, parece afectar ya a cualquier actividad y ámbito: si originariamente la intensificación era un fenómeno propio del sector industrial, hoy es innegable su propagación en los servicios, donde se aplican diversas estrategias que apuntan a racionalizarlos y estandarizarlos para ajustar los tiempos de ejecución. De igual forma, es una lógica que se ha desplazado desde el sector privado hacia el estatal a partir de las denominadas políticas de "modernización", que buscan promover la racionalización de estructuras y de procedimientos en el Estado, la evaluación por resultados (cuantitativos) y el incremento de la productividad de los empleados públicos.

Pero el ahorro de tiempo, la velocidad alcanzada y el desenfrenado ritmo que parecen imponer algunos procesos de trabajo se consigue a un alto costo, que pagan los propios trabajadores: el deterioro de su salud. De esta manera, la fatiga, las enfermedades y el sufrimiento psíquico son el precio que conllevan estas prácticas cada vez más difundidas. Como consecuencia, es necesario plantear con toda contundencia la pregunta por el impacto que pueden tener estos procesos de trabajo para la salud de los trabajadores. Esto resulta aún más importante si se tiene en cuenta que la relación entre intensificación y condiciones de trabajo es con frecuencia desconocida por los propios trabajadores y que no aparece en la agenda de los sindicatos argentinos como un tema prioritario.

En el caso de la investigación en la clínica privada hemos analizado el problema de las exigencias emocionales. Al respecto mostramos que la naturaleza de las actividades que allí se desarrollan - relacionadas con la preservación de la vida humana y el cuidado de personas en situación de enfermedad - expone a los trabajadores a condiciones muy particulares. En este sentido, deben movilizar no solo funciones físicas, sino, sobre todo, poner en juego capacidades mentales, emocionales y psíquicas de primera importancia. El sufrimiento, el dolor e incluso la muerte son aspectos recurrentes en su proceso de trabajo y, por eso, las exigencias emocionales suelen alcanzar un nivel altísimo para estos trabajadores, con los consecuentes problemas de salud que ello puede generarles.

Este fenómeno detectado en la clínica privada también puede ser vinculado con tendencias más generales, propias del régimen de acumulación vigente, en el que la dimensión emocional de los trabajadores juega un papel significativo en la creación y acumulación de valor. En todos los ámbitos, pero en particular en el sector servicios, las empresas demandan cada vez más 
que los trabajadores brinden un compromiso íntegro, que abarca no solo el despliegue de sus calificaciones estrictamente técnicas, sino también de una serie de sentimientos y emociones que deben sostener para alcanzar los objetivos prescriptos (satisfacción del cliente, aumento en las ventas, etcétera) ${ }^{11}$.

Así, el trabajo emocional es parte inherente de diversas actividades, tales como la educación, el cuidado (de niños, ancianos), el turismo, las ventas (presenciales y telefónicas), el cuidado de la salud, las industrias culturales y el entretenimiento, los servicios financieros, entre otras. En todos los casos, la eficacia productiva se alcanza por medio de una actuación específica, de dramatizaciones estandarizadas en la interacción con el cliente, de la capacidad para generarle emociones positivas y una experiencia de consumo satisfactoria. Asimismo, y con cierta frecuencia, la eficacia productiva implica atemperar el enojo del cliente, responder sus dudas, recomponer su confianza y obturar demandas que perjudiquen a la empresa.

Como decíamos antes, lo alarmante es que tanto los procesos de intensificación como las exigencias emocionales, así como sus implicaciones para la salud de los trabajadores, permanecen en gran medida invisibilizados y no son reconocidos como fenómenos problemáticos por los empleadores (a veces tampoco por los propios trabajadores). Pero ambos derivan de las exigencias que plantean los procesos de trabajo actuales y las metas de productividad que fijan las organizaciones.

Asimismo, son cuestiones que suelen relegarse al ámbito individual, negando la dimensión colectiva de estos problemas que afectan cada vez más a los trabajadores y que comienzan a ser transversales a distintas actividades productivas. En este sentido, es importante considerarlos como síntomas de procesos más amplios, que también deben ser incluidos en el análisis para comprenderlos en su totalidad. Para ello es necesario que los estudios sobre riesgos laborales progresivamente amplíen su foco, sofistiquen sus herramientas de análisis y en sus argumentos incluyan toda la serie de mediaciones que conectan los problemas de salud específicos de los trabajadores con aquellos procesos sociales y económicos más amplios de su tiempo.

11 Para controlar y verificar que estas capacidades emocionales son efectivamente desplegadas por los trabajadores, las empresas suelen recurrir a encuestas donde los clientes evalúan el trabajo o servicio recibido, donde opinan sobre el desempeño del trabajador y sobre el trato recibido. En otros casos, se suelen grabar las interacciones, como sucede en los call centers (centros de atención telefónica), para que los supervisores puedan evaluar de primera mano cómo se comportan los trabajadores con los clientes. 


\section{Referencias bibliográficas}

Agencia Europea para la Seguridad y la Salud en el Trabajo (2003). Cómo abordar los problemas psicosociales y reducir el estrés relacionado con el trabajo. Luxemburgo: Oficina de Publicaciones Oficiales de las Comunidades Europeas.

Antunes, R. (2000). La metamorfosis en el mundo del trabajo. Nómadas, 12, pp. 28-37.

Bernard, S. (2005). Le temps de l'activité de la caissière: entre logique productive et logique de service. Sociologie du travail, 47, pp. 170-187.

Blanco Gil, J. (1989). La necesaria rearticulación del conocimiento epidemiológico; el problema de la práctica-teórica y la práctica-práctica. Investigación y Educación en Enfermeria, 7(2), pp. 61-71.

Coriat, B. (1991). El taller y el cronómetro. Ensayo sobre el taylorismo, el fordismo y la producción en masa. Madrid: Siglo XXI.

Coutrot, T. y C. Mermilliod (2010). Les risques psychosociaux au travail: les indicateurs disponibles. Dares Analyses, 81, pp. 1-11.

De la Garza Toledo, E. (2013). Trabajo no clásico y flexibilidad. Caderno CRH, 26(68), pp. 315- 330 .

Esquivel, V. y F. Pereyra (2017). Las condiciones laborales de las y los trabajadores del cuidado en Argentina: Reflexiones en base al análisis de tres ocupaciones seleccionadas. Trabajo y Sociedad, 28, pp. 55-82.

Galeano, P. y P. Ruiz (2017). Exigencias emocionales y trabajo de cuidado. En: J. C. Neffa y M. L. Henry (coords.). ¿Quién cuida a los que cuidan? Los riesgos psicosociales en el trabajo en los establecimientos privados de salud. La Plata: Facultad de Ciencias Económicas Universidad Nacional de La Plata. Instituto de Investigaciones Administrativas.

Gollac, M. y S. Volkoff(1996). Citius, altius, fortius. L'intensification du travail. Actes de la Recherche en Sciences Sociales, 114, pp. 54-67.

Gollac, M. (2005). L'intensité du travail. Formes et effets. Revue économique, 56(2), pp. 195-216.

Gollac, M. (coord.) (2011). Mesurer les facteurs psychosociaux de risque au travail pour les maîtriser. París: Ministère du Travail, de l'Emploi et de la Santé.

Gollac, M. (2012). Les risques psychosociaux au travail : d'une « question de société » à des questions scientifiques. Introduction. Travail et emploi, 129, p. 5-10.

Gracia, E.; I. Martínez; M. Salanova y C. Nogareda (2007). El trabajo emocional concepto y prevención. Nota Técnica de Prevención $n .^{\circ}$ 720. Madrid: Instituto Nacional de Seguridad e Higiene en el Trabajo. Ministerio de Trabajo y Asuntos Sociales de España.

Green, F. (2004). Why has work effort become more intense? Industrial Relations, 43(4), pp. 709-741.

Henry, M. L. (2016). La encuesta como herramienta de recolección de datos sobre riesgos psicosociales. Características y balance metodológico del relevamiento realizado entre trabajadores la ANSES. Ponencia presentada en las IX Jornadas de Sociología de la UNLP, 5-7 de diciembre, La Plata.

Henry, M. L. (2017). La intensificación del trabajo como factor de riesgo psicosocial en las actividades de servicios. Ponencia presentada en el $13^{\circ}$ Congreso Nacional de Estudios del Trabajo, 2-4 de agosto, Buenos Aires.

INRS (2013). Évaluer les facteurs de risques psychosociaux: l'outil RPS-DU. París: Institut national de recherche et de sécurité. 
INSHT (2010). Factores y riesgos psicosociales, formas, consecuencias, medidas y buenas prácticas. Instituto Nacional de Seguridad e Higiene en el Trabajo. Madrid: Ministerio de Empleo y Seguridad Social.

Laurell, A. C. (1978). Proceso de trabajo y salud. Cuadernos Políticos, 17, pp. 59-79.

Laurell, C. A. (1993). La construcción teórico-metodológica de la investigación sobre salud de los trabajadores. En: C. A. Laurell (coord.). Para la investigación sobre la salud de los trabajadores. Washington: Organización Panamericana de la Salud (OPS), pp. 135-166.

Laurell, A. C. (1989). Social analysis of collective health in Latin America. Social Science \& Medicine, 28(11), pp. 1183-1191.

Laurell, C. A. y M. Noriega (1987). Trabajo y salud en SICARTSA. México: Sección 271 del Sindicato Minero, Sindicato Independiente de Trabajadores de la UAM (SITUAM) y Maestría en Medicina Social, UAM-X.

Martínez Íñigo, D. (2001). Evolución del concepto de trabajo emocional: dimensiones, antecedentes y consecuentes. Una revisión teórica. Revista de Psicología del Trabajo y de las Organizaciones, 17(2), pp. 131-153.

Ministerio de la Protección Social (2010). Batería de instrumentos para la evaluación de factores de riesgo psicosocial. Bogotá: Ministerio de la Protección Social.

Ministerio de Salud de Chile (2013). Protocolo de vigilancia de riesgos psicosociales en el trabajo. Santiago de Chile: Departamento de Salud Ocupacional del Ministerio de Salud de Chile.

Moreno Jiménez, B. (2011). Factores y riesgos laborales psicosociales: conceptualización, historia y cambios actuales. Medicina y Seguridad del Trabajo, 57, pp. 4-19.

Neffa, J. C. (1990). Proceso de trabajo y economía de tiempo. Contribución al análisis crítico del pensamiento de Karl Marx, Frederick W. Taylor y Henry Ford. Buenos Aires: Ed. CREDAL/Humanitas.

Neffa, J. C. (2015). Los riesgos psicosociales en el trabajo: contribución a su estudio. Ciudad Autónoma de Buenos Aires: CEIL/CONICET; Corrientes: UNNE, Facultad de Ciencias Económicas; La Plata: UNLP, Facultad de Ciencias Económicas; Moreno: Universidad Nacional de Moreno; CABA: UMET/CITRA.

Neffa, J. C., (coord.) (2017). Una reforma que no resuelve los problemas de los riesgos del trabajo en Argentina: debate interdisciplinario en la UMET. Buenos Aires: Universidad Metropolitana para la Educación y el Trabajo.

Neffa, J. C. y M. L. Henry (coords.) (2017). ¿Quién cuida a los que cuidan? Los riesgos psicosociales en el trabajo en los establecimientos privados de salud. La Plata: Facultad de Ciencias Económicas Universidad Nacional de La Plata. Instituto de Investigaciones Administrativas.

Neffa, J. C.; S. Korinfeld y M. L. Henry (coords.) (2017). Trabajo y salud en puestos de atención al público: una investigación sobre riesgos psicosociales en el trabajo en ANSES. Buenos Aires: SECASFPI.

Noriega, M. (1993). Organización laboral, exigencias y enfermedad. En: C. A. Laurell (coord.). Para la investigación sobre la salud de los trabajadores. Washington: Organización Panamericana de la Salud (OPS), pp. 167-187.

OIT (2016). Boletín Internacional de Investigación Sindical. Riesgos psicosociales, estrés $y$ violencia en el mundo del trabajo, 8(1-2).

Vogel, L. (2002). La organización del trabajo un ámbito decisivo en la lucha contra los sufrimientos psicológicos. Revista de Relaciones Laborales, 1, pp. 93-102. 